\title{
New Molecular Insight into Mechanism of Evolution of Mammalian Synthetic Prions
}

\author{
Natallia Makarava, ${ }^{{ }^{\dagger}}$ Regina Savtchenko, ${ }^{* \dagger}$ Irina Alexeeva,${ }^{\ddagger}$ Robert G. Rohwer, ${ }^{\ddagger \S}$ and Ilia V. Baskakov ${ }^{\star \dagger}$ \\ From the Center for Biomedical Engineering and Technology, ${ }^{*}$ and the Departments of Anatomy and Neurobiology, ${ }^{\dagger}$ and Neurology, ${ }^{\S}$ University of Maryland \\ School of Medicine, Baltimore; and the Medical Research Service, ${ }^{\ddagger}$ Veterans Affairs Medical Center, University of Maryland, Baltimore, Maryland
}

Accepted for publication November 19, 2015.

Address correspondence to Ilia V. Baskakov, Ph.D., Center for Biomedical Engineering and Technology, University of Maryland, Baltimore, $111 \mathrm{~S}$. Penn St., Baltimore, MD 21201. E-mail: baskakov@umaryland.edu.

\begin{abstract}
Previous studies established that transmissible prion diseases could be induced by in vitro-produced recombinant prion protein (PrP) fibrils with structures that are fundamentally different from that of authentic PrP scrapie isoform ( $\mathrm{Pr} \mathrm{P}^{\mathrm{Sc}}$ ). To explain evolution of synthetic prions, a new mechanism referred to as deformed templating was introduced. Here, we asked whether an increase in expression level of the cellular form of $\operatorname{PrP}\left(\mathrm{PrP}^{\mathrm{C}}\right)$ speeds up the evolution of synthetic strains in vivo. We found that in transgenic mice that overexpress hamster $\operatorname{PrP}^{C}, \operatorname{PrP}^{C}$ overexpression accelerated recombinant PrP fibril-induced conversion of $\operatorname{PrP}^{\mathrm{C}}$ to the abnormal proteinase K-resistant state, referred to as atypical PrPres, which was the first product of $\operatorname{PrP}^{C}$ misfolding in vivo. However, overexpression of $\operatorname{PrP}^{C}$ did not facilitate the second step of synthetic strain evolution-transition from atypical PrPres to $\operatorname{PrP}^{\mathrm{Sc}}$, which is attributed to the stochastic nature of rare deformed templating events. In addition, the potential of atypical PrPres to interfere with replication of a short-incubation time prion strain was investigated. Atypical PrPres was found to interfere strongly with replication of $263 \mathrm{~K}$ in vitro; however, it did not delay prion disease in animals. The rate of deformed templating does not depend on the concentration of substrate and is hence more likely to be controlled by the intrinsic rate of conformational errors in templating alternative self-propagating states. (Am J Pathol 2016, 186: 1006-1014; http://dx.doi.org/10.1016/j.ajpath.2015.11.013)
\end{abstract}

Prion diseases, or transmissible spongiform encephalopathies (TSEs), are fatal neurodegenerative disorders that can arise spontaneously, be inherited, or be acquired through transmission. ${ }^{1}$ The diversity in the cause of prion diseases can be explained by three mechanisms. Spontaneous TSE arises from the misfolding and aggregation of the normal, cellular isoform of the prion protein, $\operatorname{PrP}^{\mathrm{C}}$, into the diseaseassociated infectious scrapie isoform, $\mathrm{PrP}^{\mathrm{Sc}}$, and thus, underlies the sporadic forms of prion diseases. ${ }^{2}$ Second, single point mutations or truncations in the PRNP gene cause inherited prion diseases via facilitating misfolding of $\operatorname{PrP}^{\mathrm{C}}$. $3-5$ Third, in prion diseases acquired through the transmission, $\mathrm{PrP}^{\mathrm{Sc}}$ seeds initiate prion replication by recruiting host $\mathrm{PrP}^{\mathrm{C}}$. According to the conventional view of template-assisted replication mechanisms, prions replicate with high fidelity; ie, the folding pattern of a newly formed $\mathrm{PrP}^{\mathrm{Sc}}$ accurately reproduces that of the $\mathrm{PrP}^{\mathrm{Sc}}$ template. ${ }^{2}$

Recent studies introduced a new mechanism, referred to as deformed templating, according to which transmissible prion disease can be induced by $\operatorname{PrP}$ amyloid fibrils with structures that are fundamentally different from that of $\mathrm{PrP}^{\mathrm{Sc}}{ }^{6-9}$ The mechanism of deformed templating was used to explain transformation of self-replicating states during evolution of prion strains of synthetic origin. ${ }^{8,10-12}$ Amyloid fibrils produced from recombinant Syrian hamster PrP (rPrP) in vitro lacked any detectable $\mathrm{PrP}^{\mathrm{Sc}}$ particles, yet they were able to trigger transmissible TSEs in animals on serial passaging. ${ }^{8,10}$ In another illustration of deformed templating, noninfectious fibrils with a stacked $\beta$-sheet architecture, prepared from recombinant fungal prion protein HET-s, nucleated the infectious state of HET protein with a $\beta$-solenoid structure. ${ }^{13}$ Further illustrations of the deformed templating mechanism are offered by the so-called quaking or amyloid seeding assays, in which infectious $\operatorname{PrP}^{\mathrm{Sc}}$ nucleates noninfectious self-replicating fibrillar states when exposed to

Supported by NIH grants R01 NS045585 and R01 NS074998 (I.V.B.). Disclosures: None declared. 
rPrP in vitro. ${ }^{14,15}$ In direct support of the deformed templating mechanism, molecular imaging revealed that switching between alternative folding patterns can occur within individual amyloid fibrils. ${ }^{16}$

Previous studies established a mechanistic model for genesis of authentic $\operatorname{PrP}^{\mathrm{Sc}}$ from noninfectious fibrils that involves two main steps (Figure 1) ${ }^{8,10}$ In the first step, $\mathrm{rPrP}$ fibrils trigger $\operatorname{PrP}^{\mathrm{C}}$ misfolding, a process that results in accumulation of an alternative self-replicating state, referred to as atypical PrPres. rPrP and atypical PrPres appear to be similar in structure, because both have a short, C-terminal proteinase K (PK)-resistant core. ${ }^{8,10,17}$ In the second step, formation of authentic $\mathrm{PrP}^{\mathrm{Sc}}$ is triggered via a relatively rare deformed templating event. After the first $\mathrm{PrP}^{\mathrm{Sc}}$ particles are produced through deformed templating, $\mathrm{PrP}^{\mathrm{Sc}}$ can replicate independently of atypical PrPres (Figure 1). This mechanism proposes that two barriers on a pathway from $\mathrm{rPrP}$ fibrils to $\operatorname{PrP}^{\mathrm{Sc}}$ exist. The first barrier is attributed to a change in substrate from $r \operatorname{PrP}$ to $\operatorname{PrP}^{\mathrm{C}}$. In contrast to $\operatorname{PrP}, \operatorname{PrP}^{\mathrm{C}}$ has a glycophosphatidylinositol-anchor and $\mathrm{N}$-linked glycans, two types of post-translational modifications that might constrain conformational space acquired by self-replicating states. ${ }^{18}$ The second barrier is attributable to a low rate of deformed templating, the events by which structures different from that of atypical PrPres are generated (Figure 1).

In the current study we tested whether substrate plays a role in evolution of authentic $\operatorname{PrP}^{\mathrm{Sc}}$. Would evolution of infectious $\mathrm{PrP}^{\mathrm{Sc}}$ structures be faster or more efficient in animals overexpressing $\operatorname{PrP}^{\mathrm{C}}$ ? We found that, in transgenic $(\operatorname{tg} 7)$ mice that overexpress hamster $\operatorname{PrP}^{\mathrm{C}}$ on an ablated background, ${ }^{19}$ high concentration of a substrate helped to overcome the first barrier, which is the transition from $\mathrm{rPrP}$ to $\operatorname{PrP}^{\mathrm{C}}$ as a substrate and formation of atypical PrPres. However, overexpression of $\operatorname{PrP}^{\mathrm{C}}$ did not facilitate crossing

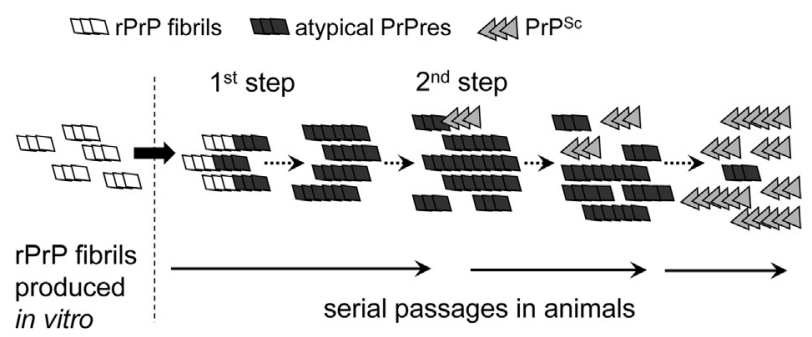

Figure 1 Schematic presentation of the mechanism, illustrating genesis of $\operatorname{PrP}{ }^{S c}$ triggered by rPrP fibrils. In a first step, $r$ PrP fibrils seeded atypical PrPres, a transmissible form of PrP that replicates silently without causing clinical disease. Replication of atypical PrPres occasionally produces $\mathrm{PrP}^{\mathrm{Sc}}$ in seeding events that appears to be rare and stochastic as described for a deformed templating mechanism. $\mathrm{PrP}^{\mathrm{Sc}}$ replicates faster than atypical PrPres and eventually replaces it during serial passages. The two forms atypical PrPres and $\mathrm{PrP}^{\mathrm{Sc}}$ can be distinguished after PK treatment via staining Western blot analyses with 3F4 and SAF-84 antibody. Because PK-resistant regions of atypical PrPres is shorter than that of PrPS , it binds only SAF-84 antibody (epitope 160 to 170), whereas $\operatorname{PrP}^{\mathrm{Sc}}$ binds both 3F4 (epitope 109 to 112) and SAF-84 antibodies. PK, proteinase K; atypical PrPres, alternative self-replicating state of prion protein; $\mathrm{PrP}^{\mathrm{Sc}}$, prion protein scrapie isoform; $\mathrm{rPrP}$, recombinant prion protein. the second barrier, which is the transition from atypical PrPres to $\mathrm{PrP}^{\mathrm{Sc}}$. This result suggests that the rate of deformed templating is not controlled by $\operatorname{PrP}^{\mathrm{C}}$ expression levels. Moreover, although $\operatorname{PrP}^{\mathrm{C}}$ overexpression did not speed up the overall rate of evolution of synthetic prions, it expanded the range of self-replicating fibrillar states generated in vitro that were capable of inducing TSEs in animals.

\section{Materials and Methods}

\section{Expression and Purification of $\mathrm{rPrP}$ and Formation of rPrP Fibrils}

Syrian hamster full-length recombinant PrP that encompass residues 23 to 231 was expressed and purified as previously described, ${ }^{20}$ with minor modifications. ${ }^{9}$ Lyophilized $\mathrm{rPrP}$ was dissolved in $5 \mathrm{mmol} / \mathrm{L}$ HEPES, pH 7.0, immediately before use. To form fibrils for inoculations, a mixture of $0.5 \mathrm{mg} / \mathrm{mL} \mathrm{rPrP}$ with $50 \mathrm{mmol} / \mathrm{L} 2$-( $N$-morpholino)ethanesulfonic acid buffer, $\mathrm{pH} 6.0$, and $2.0 \mathrm{~mol} / \mathrm{L}$ guanidine hydrochloride $(\mathrm{GdnHCl})$ was incubated at $37^{\circ} \mathrm{C}$ under continuous agitation. Amyloid formation was confirmed by thioflavin $\mathrm{T}$ fluorescence assay, epifluorescent microscopy, and electron microscopy as described previously. ${ }^{20}$ For tg7 mouse inoculations, fibrils were diluted to $0.23 \mathrm{mg} / \mathrm{mL} \mathrm{rPrP}$ and dialyzed into phosphate-buffered saline (PBS), pH 7.4. To prepare $\alpha$-rPrP for inoculating the control group, $\mathrm{rPrP}$ stock solution was diluted in PBS to a final protein concentration of $0.23 \mathrm{mg} / \mathrm{mL}$. Conformation of $\alpha-\operatorname{rPrP}$ was confirmed by circular dichroism. Syrian hamsters were inoculated with fibrils at the concentration of $0.5 \mathrm{mg} / \mathrm{mL}$ rPrP dialyzed into PBS, pH 7.4.

\section{Bioassay}

All inoculations were performed intracerebrally, under $2 \%$ $\mathrm{O}_{2} / 4$ minimum alveolar concentration isoflurane anesthesia. Each mouse or hamster received 30 or $50 \mu \mathrm{L}$ of inoculum, respectively, into the left hemisphere, approximately $3 \mathrm{~mm}$ to the left of the midline and approximately $3 \mathrm{~mm}$ anterior to a line drawn between the ears. After inoculation, animals were observed daily for disease with the use of a blind scoring protocol. For the first passage, weanling tg 7 mice or Syrian hamsters were inoculated with $\mathrm{rPrP}$ amyloid fibrils or $\alpha$-rPrP prepared as described above. Mice and hamsters were euthanized at 510 to 524 and 659 to 664 days after inoculation, respectively, without any sign of clinical disease, and their brains were removed aseptically and saved for analysis and second passage. For the second and third passages, $10 \%$ brain homogenates (BHs) prepared by homogenization in PBS, $\mathrm{pH} 7.4{ }^{11}$ were dispersed by 30 seconds of sonication immediately before inoculation. For the experiment on interference, protein misfolding cyclic amplification with partially deglycosylated substrate (dgPMCA)-derived atypical PrPres produced as described below was diluted 10-fold with PBS supplemented with $1 \%$ 
bovine serum albumin and spiked with $10^{-4}$ brain material from a terminally ill animal infected with $263 \mathrm{~K}$. As controls, 10-fold diluted dgPMCA-derived atypical PrPres or $10^{-4}$ $263 \mathrm{~K}$ in PBS with $1 \%$ bovine serum albumin was inoculated separately. This study was performed in strict accordance with the recommendations in the NIH's Guide for the Care and Use of Laboratory Animals. ${ }^{21}$ The animal protocol was approved by the Institutional Animal Care and Use Committee of the University of Maryland, Baltimore (assurance no. A32000-01; permit no. 0215002).

\section{PK Digestion, Western Blot Analysis, and Data Analysis}

For the PK digestion in sarcosyl, an aliquot of $10 \% \mathrm{BH}$ prepared as described previously ${ }^{11}$ was mixed with an equal volume of $4 \%$ sarcosyl in PBS, supplemented with $50 \mathrm{mmol} / \mathrm{L}$ Tris, $\mathrm{pH} 7.5$, and digested with $20 \mu \mathrm{g} / \mathrm{mL}$ PK (New England BioLabs, Ipswich, MA) for 30 minutes at $37^{\circ} \mathrm{C}$ with $1000 \mathrm{rpm}$ shaking with the use of a DELFIA plate shaker (Perkin Elmer, Waltham, MA) placed in an incubator at $37^{\circ} \mathrm{C}$. PK digestion was stopped by adding SDS sample buffer and heating the samples for 10 minutes in a boiling water bath. Samples were loaded onto NuPAGE $12 \%$ Bis-Tris gels, transferred to polyvinylidene difluoride membrane, and probed with $3 \mathrm{~F} 4$ or SAF- 84 antibodies.

To ensure accurate calculation of $\operatorname{PrP}^{\mathrm{Sc}}$ amount in brains of animals from second and third passage, $10 \% \mathrm{BHs}$ were dispersed by 30 seconds of sonication of $100-\mu \mathrm{L}$ aliquots in the microplate horn of Qsonica sonicator (Newton, CT), the total protein concentration was measured by Bradford Assay (Bio-Rad, Hercules, CA), and normalized before performing PK digestion. For statistical analysis, samples from the second and third passages were loaded onto the same gel and were also probed with anti- $\beta$-actin antibody (SigmaAldrich, St. Louis, MO) to verify equal gel loading. Images were captured with the use of FluorChem imager (ProteinSimple, San Jose, CA). The signal intensity was digitized for densitometry analysis with the use of AlphaView software version 4.1.3 (ProteinSimple), then normalized by $\beta$-actin signal, and statistical significance was calculated with the use of $t$-test.

\section{PMCAb}

Normal BH (10\%) from healthy hamsters was prepared as described previously ${ }^{8}$ and was used as a substrate for PMCA with beads (PMCAb). ${ }^{22}$ For the first round, $10 \mu \mathrm{L}$ of BH from inoculated animals was added to $90 \mu \mathrm{L}$ of normal BH. The standard sonication program consisted of 20 seconds of sonication pulses at approximately $150 \mathrm{~W}$ applied every 20 minutes during a 24-hour period. For each subsequent round, $10 \mu \mathrm{L}$ of the reaction from the previous round was added to $90 \mu \mathrm{L}$ of fresh substrate. Each PMCAb reaction was performed in the presence of two 3/32-inch Teflon beads (McMaster-Carr, Los Angeles, CA). To analyze production of PK-resistant PrP material in PMCAb, $10 \mu \mathrm{L}$ of sample was supplemented with $5 \mu \mathrm{L}$ of SDS and $5 \mu \mathrm{L}$ of PK to a final concentration of SDS and PK of $0.25 \%$ and $50 \mu \mathrm{g} / \mathrm{mL}$, respectively, followed by incubation at $37^{\circ} \mathrm{C}$ for 1 hour. The digestion was terminated by adding SDS-sample buffer and heating the samples for 10 minutes in a boiling water bath.

\section{dgPMCAb and Generation of Atypical PrPres}

To produce substrate for dgPMCAb, $10 \%$ normal $\mathrm{BH}$ from healthy hamsters prepared for PMCAb (see section above) was treated with peptide $\mathrm{N}$-glycosidase F (New England BioLabs; glycerol-free) as follows. After preclearance of normal $\mathrm{BH}$ at $500 \times g$ for 2 minutes, $1500 \mathrm{U} / \mathrm{mL}$ peptide $\mathrm{N}$-glycosidase F was added to the supernatant fluid, and the reaction was incubated on a rotator at $37^{\circ} \mathrm{C}$ for 5 hours. The resulting substrate was used in dgPMCAb with sonication conditions described for PMCAb.

To produce atypical PrPres for in vitro and in vivo interference study, dgPMCA substrate was treated with RNase A (Sigma-Aldrich; catalog no. R4875) for 1 hour at $37^{\circ} \mathrm{C}$, as described previously, ${ }^{23}$ and seeded with $10^{-9}$ brain material of an animal from the second passage of synthetic strain S05, which contained predominantly atypical PrPres. ${ }^{10}$ dgPMCAb was performed for 18 serial rounds with 10 -fold dilution between rounds. The absence of $\mathrm{PrP}^{\mathrm{Sc}}$ in the final products was confirmed by failure to amplify in six rounds of PMCAb (data not shown).

\section{Histopathologic Study}

Formalin-fixed brain halves divided at the midline were processed for immunohistochemistry staining with the use of the mouse monoclonal anti-PrP antibody 3F4 (dilution 1:1000; BioLegend, San Diego, CA). Blocks were treated in formic acid (96\%) before being embedded in paraffin. Epitope recovery was performed by 15 minutes of hydrated autoclaving at $121^{\circ} \mathrm{C}$ in trisodium citrate buffer, $\mathrm{pH} 6.0$, with $0.05 \%$ Tween 20 , followed by 5 minutes in $88 \%$ formic acid.

\section{Results}

\section{M GdnHCl rPrP Fibrils Induce Formation of Atypical PrPres in tg7 Mice}

In previous studies, fibrils produced in vitro with the use of full-length Syrian hamster rPrP in different solvent conditions had different capacities for seeding misfolding of $\mathrm{PrP}^{\mathrm{C}}$ in vivo, arguing that the efficiency in triggering authentic $\mathrm{PrP}^{\mathrm{Sc}}$ is controlled by the conformation of rPrP fibrils. ${ }^{10} \mathrm{On}$ inoculation of Syrian hamsters, fibrils formed in $0.5 \mathrm{~mol} / \mathrm{L}$ $\mathrm{GdnHCl}$ (referred to as $0.5 \mathrm{M}$ fibrils) induced atypical PrPres in six of seven animals in the first passage and caused transmissible prion disease in the second passage. ${ }^{10}$ In contrast, no signs of PK-resistant products were detected by Western blot analysis or serial PMCAb assay in animals 


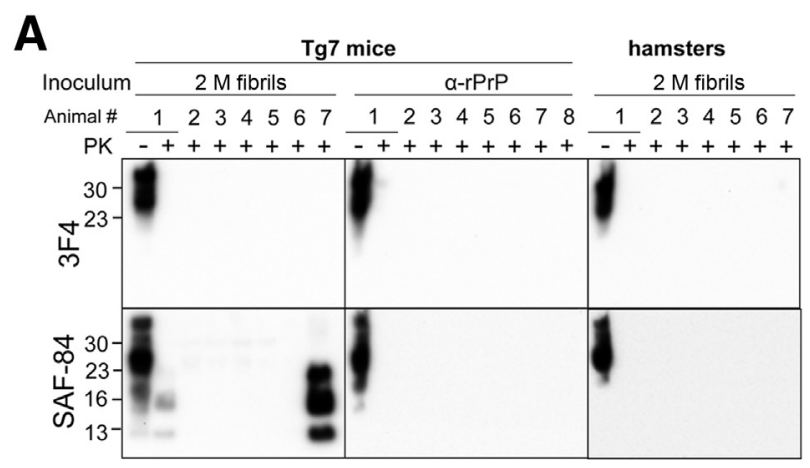

B

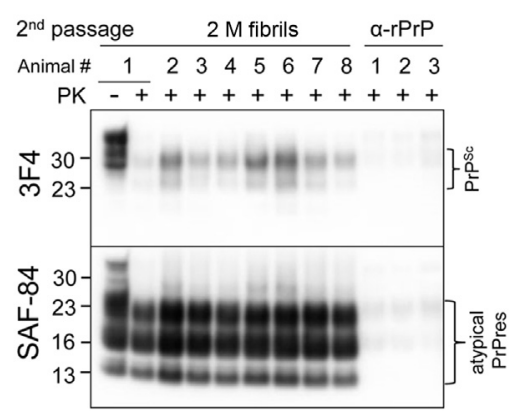

Figure 2 Bioassay of $2 \mathrm{M} \mathrm{rPrP}$ fibrils in tg7 mice. A: Western blot analyses of brain homogenates from either $\mathrm{tg} 7$ mice inoculated with $2 \mathrm{M} \mathrm{rPrP}$ fibrils or $\alpha$-rPrP or Syrian hamsters inoculated with $2 \mathrm{M}$ rPrP fibrils and stained with $3 \mathrm{~F} 4$ or SAF- 84 antibody. The inoculation of fibril material is referred to as first passage. B: Western blot analyses of brain homogenates from the second passage of $2 \mathrm{M} \mathrm{rPrP}$ fibrils or $\alpha-\mathrm{rPrP}$ in tg7 mice stained with $3 \mathrm{~F} 4$ or SAF-84 antibody. PK-resistant bands at 23,16 , and $13 \mathrm{kDa}$ represent di-, mono-, and unglycosylated atypical PrPres, respectively. PK, proteinase K; atypical PrPres, alternative self-replicating state of prion protein; $\mathrm{PrP}^{\mathrm{Sc}}$, prion protein scrapie isoform; $r \mathrm{PrP}$, recombinant prion protein; $\mathrm{tg}$, transgenic.

( 0 of 7 animals) inoculated by the fibrils formed in $2 \mathrm{~mol} / \mathrm{L}$ GdnHCl (2 $\mathrm{M}$ fibrils ${ }^{10}$ ) (Figure 2A).

The current work asked the question whether an increase in expression level of $\operatorname{PrP}^{\mathrm{C}}$ helps to trigger TSEs by $2 \mathrm{M}$ fibrils that would otherwise fail to induce disease in wildtype animals. $\operatorname{Tg} 7$ mice that express $\operatorname{PrP}^{\mathrm{C}}$ at a level approximately 3.5 -fold higher than that in hamster ${ }^{19}$ were inoculated with $2 \mathrm{M}$ fibrils or $\mathrm{rPrP}$ folded into a normal $\alpha$-helical conformation $(\alpha$-rPrP). Two of seven tg7 mice inoculated with $2 \mathrm{M}$ fibrils produced atypical PrPres (Figure 2A and Table 1). Discrimination between atypical PrPres and $\operatorname{PrP}^{\mathrm{Sc}}$ is possible because PK-resistant fragments of atypical PrPres encompass residues approximately 150 to 231 and is immunoreactive with SAF-84 (epitope 160 to 170 ) but not 3F4 antibody (epitope 109 to 112). ${ }^{8,24}$ PK-resistant core of $\mathrm{PrP}^{\mathrm{Sc}}$ encompasses residues approximately 90 to 231 and can be detected by both $3 \mathrm{~F} 4$ and SAF-84. As in previous studies, ${ }^{8,10,12}$ atypical PrPres consisted of three bands of 23,16 , and $13 \mathrm{kDa}$, corresponding to di, mono-, and unglycosylated C-terminal fragments, respectively, which are immunoreactive with SAF-84 but not 3F4 antibody (Figure 2A). No PK-resistant PrP material was found in the control tg7 group inoculation with $\alpha$-rPrP (0 of 8 ) or the hamster group inoculated with $2 \mathrm{M}$ fibrils (0 of 8) (Figure 2A and Table 1). $\mathrm{PrP}^{\mathrm{Sc}}$ was not detected in any of these three animal groups as probed by $3 \mathrm{~F} 4$ antibody (Figure 2A). In summary, although $2 \mathrm{M}$ fibrils were unable to trigger $\operatorname{PrP}^{\mathrm{C}}$ misfolding in wild-type animals, they induced formation of atypical PrPres in two of seven animals overexpressing hamster $\operatorname{PrP}^{\mathrm{C}}$. This result suggests that an increase in $\mathrm{PrP}^{\mathrm{C}}$ expression level facilitates crossing of the first barrier, ie, a transition from rPrP fibrils to atypical PrPres.

In previous experiments, wild-type animals had already produced subclinical levels of $\operatorname{PrP}^{\mathrm{Sc}}$ by the first passage on inoculation of $0.5 \mathrm{M}$ fibrils. ${ }^{10} \mathrm{In}$ contrast, no $\mathrm{PrP}^{\mathrm{Sc}}$ was found in the first passage of $2 \mathrm{M}$ fibrils in $\operatorname{tg} 7$ mice, either by Western blot analysis (Figure 2A) or serial PMCAb (data not shown). Lack of detectible $\mathrm{PrP}^{\mathrm{Sc}}$ in tg7 mice suggests that overexpression of $\operatorname{PrP}^{\mathrm{C}}$ does not help to speed up the transition from atypical PrPres to $\operatorname{PrP}^{\mathrm{Sc}}$, ie, crossing of the second barrier. Alternatively, unlike atypical PrPres produced by 0.5 M fibrils, atypical PrPres generated by $2 \mathrm{M}$ fibrils might be unable to produce authentic $\mathrm{PrP}^{\mathrm{Sc}}$. To address this question, a second passage was conducted in $\operatorname{tg} 7$ mice.

Table 1 Bioassay of rPrP Amyloid Fibrils

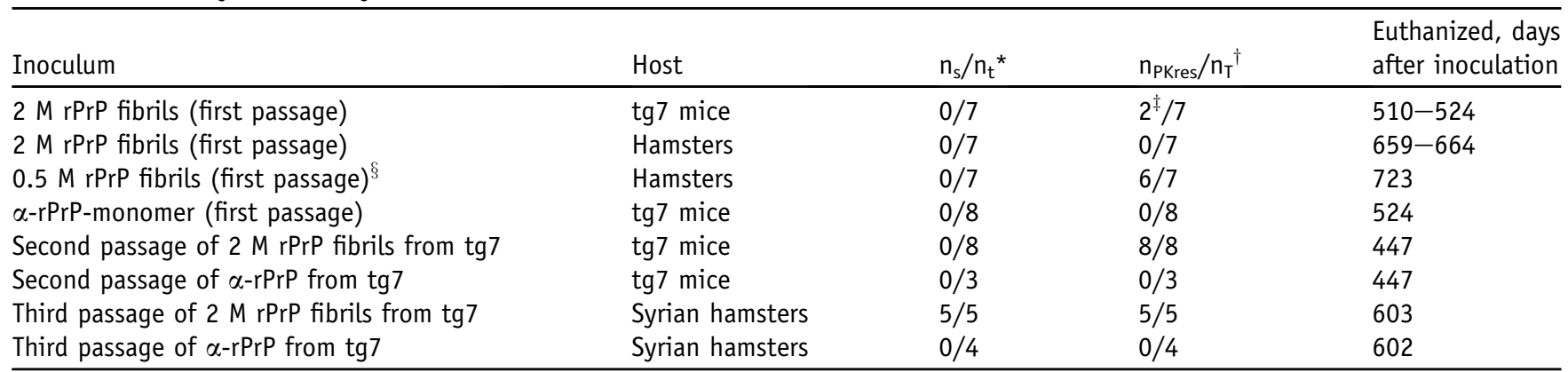

*Number of animals with clinical signs over the total number of animals survived to the end of the experiment.

†Number of animals with PK-resistant PrP in BHs over the total number of animals survived to the end of the experiment.

${ }^{\ddagger}$ In two BHs atypical PrPres were found by PK digestion assay.

${ }^{\S}$ Data are from Makarava et al. ${ }^{10}$

$\mathrm{BH}$, brain homogenate; PK, proteinase K; atypical PrPres, alternative self-replicating state of prion protein; $r$ PrP, recombinant prion protein; $\mathrm{tg}$, transgenic. 
Overexpression of $\operatorname{PrP}{ }^{C}$ Does Not Facilitate Transition from Atypical PrPres to $\operatorname{PrP}^{\mathrm{Sc}}$

Brain materials from one $\operatorname{tg} 7$ mouse positive for atypical PrPres and one control tg7 mouse inoculated with $\alpha$-rPrP were used to produce second passage in $\operatorname{tg} 7$ mice. All eight animals from the second passage of $2 \mathrm{M}$ fibrils showed substantial amounts of atypical PrPres in their brains, but they did not develop TSE signs for up to 447 days after inoculation (Figure 2B and Table 1). In agreement with the model presented in Figure 1, small amounts of $\operatorname{PrP}^{\mathrm{Sc}}$ were detected in all brains by Western blot analysis, in addition to atypical PrPres (Figure 2B). No atypical PrPres or $\mathrm{PrP}^{\mathrm{Sc}}$ was found in the second passage of $\alpha$-rPrP (Figure 2B). In good agreement with previous studies, ${ }^{8,10,12}$ atypical PrPres was found to be transmissible, and its serial passaging resulted in formation of $\operatorname{PrP}^{\mathrm{Sc}}$. However, because $\operatorname{PrP}^{\mathrm{Sc}}$ appeared only in the second passage, overexpression of $\operatorname{PrP}^{\mathrm{C}}$ did not seem to affect the rate of deformed templating events that led to $\operatorname{PrP}^{\mathrm{Sc}}$.

Further evidence that the high level of $\operatorname{PrP}^{\mathrm{C}}$ expression does not speed up deformed templating events was found in a comparison of the amounts of atypical PrPres and $\mathrm{PrP}^{\mathrm{Sc}}$ in the following animal groups: $\mathrm{Tg} 7$ mice from second passage of $2 \mathrm{M}$ fibrils and asymptomatic hamsters from the second passage of $0.5 \mathrm{M}$ fibrils. ${ }^{10}$ The amounts of atypical PrPres were much higher in $\operatorname{tg} 7$ mice than in hamsters (Figure 3), which is consistent with higher expression levels of $\mathrm{PrP}^{\mathrm{C}}$ in tg 7 mice. However, the amounts of $\operatorname{PrP}^{\mathrm{Sc}}$ in the second passage of $2 \mathrm{M}$ fibrils were approximately the same as in the asymptomatic animals from the second passage of $0.5 \mathrm{M}$ fibrils (Figure 3). The size of the PK-resistant bands and the glycoform ratio were similar in atypical PrPres generated from

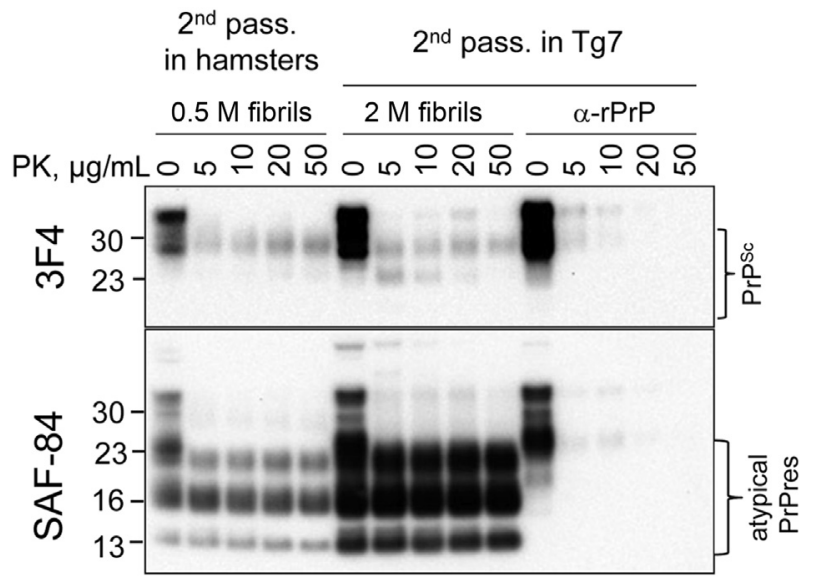

Figure 3 Comparison of $\mathrm{PrP}^{\mathrm{Sc}}$ and atypical PrPres amounts in animals from the second passage. Western blot analyses of brain homogenates from the second passage of $0.5 \mathrm{M} \mathrm{rPrP}$ fibrils in Syrian hamsters or the second passage of $2 \mathrm{M} r \operatorname{PrP}$ fibrils or $\alpha-\mathrm{rPrP}$ in tg7 mice. Brain materials were treated with increasing concentrations of PK as indicated and stained with 3F4 or SAF-84 antibody. PK, proteinase K; atypical PrPres, alternative selfreplicating state of prion protein; $\mathrm{PrP}^{\mathrm{Sc}}$, prion protein scrapie isoform; $\mathrm{rPrP}$, recombinant prion protein; $\mathrm{tg}$, transgenic.

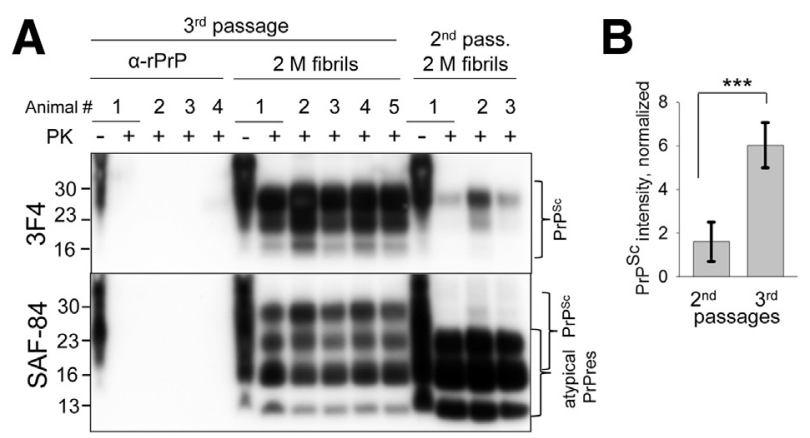

Figure 4 Third passage of $2 M$ rPrP fibrils or $\alpha$-rPrP. A: Western blot analyses of brain homogenates from the third passage of $2 \mathrm{M} \mathrm{rPrP}$ fibrils or $\alpha-r P r P$ conducted in Syrian hamsters. Analysis of brain materials from the second passage of $2 \mathrm{M} \mathrm{rPrP}$ fibrils is provided for comparison. Western blot analyses were stained with $3 \mathrm{~F} 4$ or SAF-84 antibody, as indicated. $3 \mathrm{~F} 4$ reacts only with $\mathrm{PrP}{ }^{\mathrm{Sc}}$, whereas SAF-84 stains both $\mathrm{PrP}^{\mathrm{SC}}$ and atypical PrPres. B: Quantification and statistical analysis of $\mathrm{PrP}^{\mathrm{Sc}}$ in animal brains from the second and third passages of $2 \mathrm{M}$ fibrils. For calculating means $\pm S D$, the Western blot analysis presented in Supplemental Figure S1 (eight animals form the second and five animals from the third passages) stained with 3F4 antibody was used. PrP ${ }^{\mathrm{Sc}}$ signal intensity was normalized by $\beta$-actin signal intensity. ${ }^{* * *} P<0.0005$. $\mathrm{PK}$, proteinase $\mathrm{K}$; atypical PrPres, alternative self-replicating state of prion protein; $\mathrm{PrP}^{\mathrm{Sc}}$, prion protein scrapie isoform; $\mathrm{rPrP}$, recombinant prion protein.

$0.5 \mathrm{M}$ fibrils in hamsters and from $2 \mathrm{M}$ fibrils in tg7 mice. In summary, these results demonstrate that overexpression of $\operatorname{PrP}^{\mathrm{C}}$ accelerates accumulation of atypical PrPres but does not help crossing the second barrier, ie, the transition from atypical PrPres to $\operatorname{PrP}^{\mathrm{Sc}}$.

\section{Third Passage of 2 M Fibrils Leads to Accumulation of $\mathrm{PrP}^{\mathrm{Sc}}$ and Clinical Disease}

According to the model in Figure 1, atypical PrPres and $\mathrm{PrP}^{\mathrm{Sc}}$ are two alternative self-propagating forms that compete for the same substrate. In previous studies, $\operatorname{PrP}^{\mathrm{Sc}}$ outcompeted and replaced atypical PrPres during serial passaging. ${ }^{8,10}$ To test whether this is the case for transmissible materials produced from $2 \mathrm{M}$ fibrils and to allow more time for this process to take place, the third passage of $2 \mathrm{M}$ fibrils was performed in Syrian hamsters instead of $\operatorname{tg} 7$ mice. Atypical PrPres continued to propagate in the third passage, although its amount declined compared with the amounts in the second passage (Figure 4A). Nevertheless, this highlights the highly transmissible nature of this state. Remarkably, the amounts of $\operatorname{PrP}^{\mathrm{Sc}}$ increased significantly in the third passage compared with the group from the second passage (Figure 4, A and B, and Supplemental Figure S1). Moreover, clinical disease was observed at approximately 500 days after inoculation (Table 1). Consistent with slow disease progression in previous studies on hamster synthetic strains, ${ }^{8,10}$ the clinical disease here progressed slowly, and animals approached terminal stages approximately 100 days after the first signs. Staining of brain slices with 3F4 revealed large plaques in the subpial and subependymal region (Figure 5), which is a hallmark of hamster synthetic 


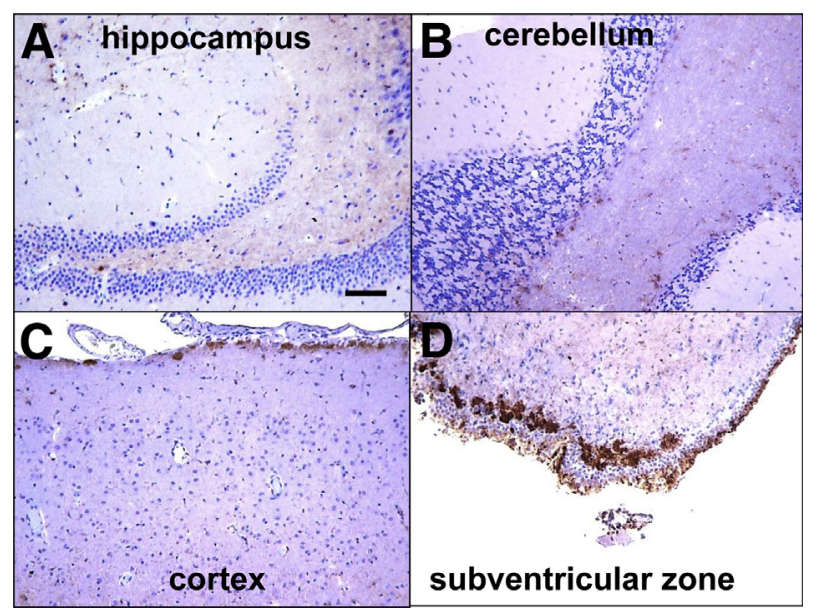

Figure 5 PrP depositions in hamsters from the third passage. A-D: PrP immunoreactivity stained with $3 \mathrm{~F} 4$ antibody in hippocampus (A), cerebellum (B), cortex (C), and subventricular zone (D) in animals from the third passage of $2 \mathrm{M}$ fibrils. Scale bar $=200 \mu \mathrm{m}$. PrP, prion protein.

strains (Figure 5). ${ }^{8-10}$ Diffuse, synaptic PrP immunoreactivity was observed in multiple brain areas, including hippocampus and cerebellum (Figure 5). In summary, similar to the previous studies on synthetic strains S05 and LOTSS, ${ }^{8,10}$ serial passaging led to accumulation of $\operatorname{PrP}^{\mathrm{Sc}}$, a decline in the amounts of atypical PrPres, and a development of clinical prion disease.

\section{Interference between Atypical PrPres and $\operatorname{PrP} \mathrm{Sc}$}

Considering the competing nature of atypical PrPres and $\mathrm{PrP}^{\mathrm{Sc}}$ and because replication of atypical PrPres alone does not cause the disease, ${ }^{8,10,12}$ we were interested in testing whether atypical PrPres material could be used to suppress replication of $\mathrm{PrP}^{\mathrm{Sc}}$ and to delay or prevent prion disease. To prepare atypical PrPres, brain-derived material from an asymptomatic S05 animal of the second passage with high concentration of atypical PrPres ${ }^{10}$ was used to seed a dgPMCAb reaction (the PMCAb format that uses partially deglycosylated $\operatorname{PrP}^{\mathrm{C}}$ as a substrate ${ }^{17}$ ). To make sure that the inoculum lacks even miniscule amounts of $\operatorname{PrP}^{\mathrm{Sc}}, 18$ serial dgPMCAb rounds were conducted with RNase-treated substrate (see Materials and Methods).

In support of our hypothesis, in vitro experiments revealed that the presence of atypical PrPres interfered with the amplification of $\operatorname{PrP}^{\mathrm{Sc}}$ of hamster-adapted scrapie strain $263 \mathrm{~K}$ in PMCAb (Figure 6). Notably, in dgPMCAb that uses partially deglycosylated $\operatorname{PrP}^{\mathrm{C}}$ as a substrate,${ }^{17}$ atypical PrPres was amplifiable regardless of whether $263 \mathrm{~K} \mathrm{PrP}^{\mathrm{Sc}}$ was present in the reaction mixture (Figure 6).

Next, we tested the interference effect with the use of animal bioassay. Hamsters were inoculated with $10^{-4}$ diluted $263 \mathrm{~K}$ brain-derived material alone, $10^{-4}$ diluted $263 \mathrm{~K}$ brain-derived material mixed with dgPMCAb-derived atypical PrPres, or dgPMCAb-derived atypical PrPres. As expected, animals inoculated with $263 \mathrm{~K}$ brain material

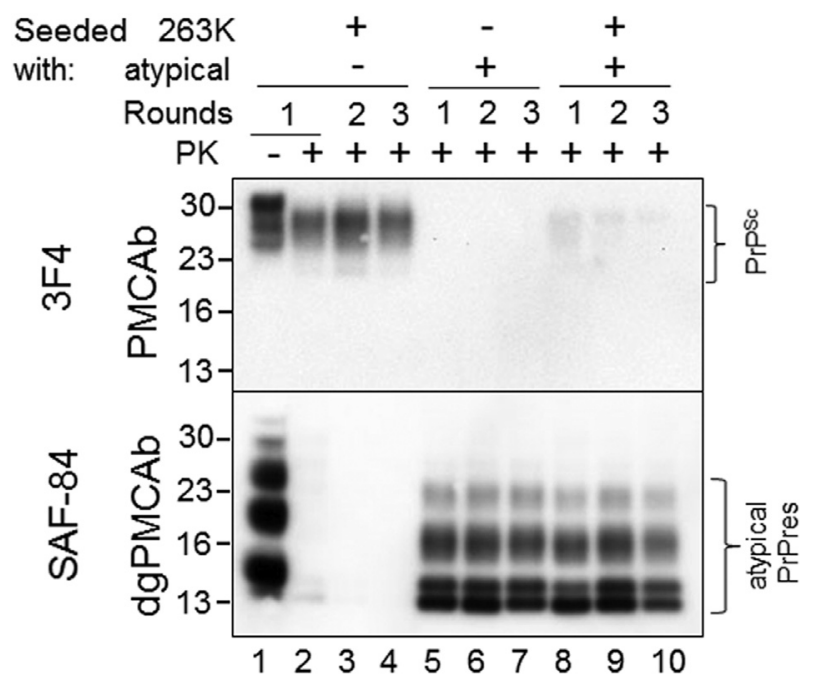

Figure 6 Competition between atypical and $\mathrm{PrP}^{\mathrm{Sc}}$ in PMCAb or dgPMCAb. Serial PMCAb or dgPMCAb reactions were seeded with $10^{-4}$ diluted $263 \mathrm{~K}$ brain material (lanes 2 to 4 ), $10 \mu \mathrm{L}$ of dgPMCAb reaction that contained atypical PrPres (lanes 5 to 7 ) or a mixture of $263 \mathrm{~K}$ and atypical PrPres (lanes 8 and 9). Three serial rounds were conducted with 10 -fold dilution between rounds. Western blot analyses were stained with $3 \mathrm{~F} 4$ or SAF-84 antibody as noted. dgPMCAb, protein misfolding cyclic amplification with beads and partially deglycosylated substrate; PK, proteinase K; PMCAb, protein misfolding cyclic amplification with beads; atypical PrPres, alternative self-replicating state of prion protein; $\operatorname{Pr} \mathrm{P}^{\mathrm{Sc}}$, prion protein scrapie isoform.

developed first clinical sings of TSE at $84 \pm 3$ days after inoculation (16 of 16) and showed a strong signal on Western blot analysis (Figure 7). Animals inoculated with a mixture of $263 \mathrm{~K}$ and atypical PrPres developed clinical diseases at $84 \pm 8$ days after inoculation (6 of 6) and showed an equally strong signal on Western blot analysis. Unfortunately, mixing atypical PrPres with $263 \mathrm{~K}$ did not delay the incubation time to disease nor slow the progression of the disease. Animals inoculated with atypical PrPres alone were asymptomatic, and no $\mathrm{PrP}^{\mathrm{Sc}}$ or atypical PrPres were detected in their brains at 116 days after inoculation

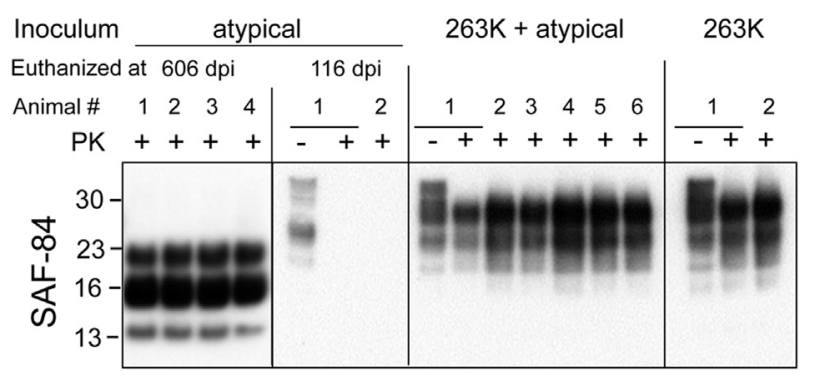

Figure 7 Bioassay for interference between atypical and $\mathrm{PrP}{ }^{\mathrm{Sc}}$. Hamsters were inoculated with $10^{-4} 263 \mathrm{~K}$ brain-derived material alone, $10^{-4}$ 263K brain-derived material mixed with dgPMCAb-derived atypical PrPres, or dgPMCAb-derived atypical PrPres alone. Animals inoculated with atypical PrPres were euthanized at 116 or 606 dpi. Western blot analyses were stained with SAF-84 antibody. dgPMCAb, protein misfolding cyclic amplification with beads and partially deglycosylated substrate; dpi, days after inoculation; PK, proteinase K; atypical PrPres, alternative self-replicating state of prion protein; $\mathrm{PrP}^{\mathrm{Sc}}$, prion protein scrapie isoform. 
(Figure 7). However, the animals inoculated with atypical PrPres did show substantial amounts of atypical PrPres in their brain, although without any clinical signs when euthanized at 606 days after inoculation (Figure 7). This result establishes that, although atypical PrPres is transmissible, it takes a long time to accumulate in vivo, and atypical PrPres does not effectively interfere with a shortincubation time strain.

\section{Discussion}

Current work provides new insight into the evolution of transmissible prion states and helps to refine the model developed from previous studies. ${ }^{8,10}$ First, we showed that although $2 \mathrm{M} r P r P$ fibrils failed to induce $\operatorname{PrP}^{\mathrm{Sc}}$ and TSE in wild-type animals, they triggered prion replication de novo in tg7 mice. Because serial transmission of $\alpha$-rPrP failed to induce TSE in $\operatorname{tg} 7$ animals, this effect appears to be fibril specific. Taken together these results illustrate that the range of self-replicating conformations generated in vitro that are capable of inducing TSEs can be expanded in animal models that overexpress $\operatorname{PrP}^{\mathrm{C}}$. Second, the current work illustrates that overexpression of $\operatorname{PrP}^{\mathrm{C}}$ helps to overcome the first barrier, ie, the transition from $r P r P$ fibrils to atypical PrPres. Because atypical PrPres is the first product of $\mathrm{PrP}^{\mathrm{C}}$ misfolding seeded by $\mathrm{rPrP}$ fibrils in vivo, the first barrier is attributable to a change in a substrate from $\operatorname{rPrP}$ to $\operatorname{PrP}^{\mathrm{C}}$. Third, overexpression of $\operatorname{PrP}^{\mathrm{C}}$ did not facilitate passing the second barrier, which is the transition from atypical PrPres to $\operatorname{PrP}^{\mathrm{Sc}}$. $\operatorname{PrP}^{\mathrm{Sc}}$ is produced in seeding events that appear to be rare and stochastic in nature and are described by a deformed templating mechanism. ${ }^{8,10}$ Current results argue that the rate of deformed templating does not depend on the expression level of a substrate but is likely to be controlled by the nature of the template, intrinsic rate of conformational errors in templating, and, possibly, the biochemical environment. We do not know whether the rate of deformed templating events depends on cofactors and/or their species specificity. If it does, cofactors might not only shape the strain-specific structure, as was shown in a previous study, ${ }^{25}$ but also regulate evolutionary processes. In summary, although the transition from rPrP to atypical PrPres happened more efficiently in $\operatorname{tg} 7$ mice, overexpression of $\operatorname{PrP}^{\mathrm{C}}$ did not speed up disease development.

Previous studies on mouse synthetic prions showed that a range of $\mathrm{PrP}^{\mathrm{Sc}}$ conformations with different incubation times to disease and disease phenotypes can be generated in mice overexpressing mouse $\operatorname{PrP}^{\mathrm{C}}$ with the use of $\mathrm{rPrP}$ fibrils produced in vitro under different solvent conditions. ${ }^{26-30} \mathrm{It}$ is not clear whether any atypical PrP states are involved in the evolution of synthetic mouse prions, because no mouse atypical PK-resistant states were described. Nevertheless, because the structures of mouse $\mathrm{rPrP}$ fibrils generated in vitro and $\mathrm{PrP}^{\mathrm{Sc}}$ were found to be fundamentally different, ${ }^{31,32}$ a transition from fibril-specific to $\mathrm{PrP}^{\mathrm{Sc}}$-specific structures should involve deformed templating, whether it occurs directly or via as yet unknown intermediate steps. In a manner similar to evolution of synthetic hamster strains, ${ }^{11}$ physical and biological properties of mouse strains changed gradually on serial passages, ${ }^{27,29,30}$ highlighting the common trends in evolution of strains of synthetic origin.

As judged from the size and position of the PK-resistant core, atypical PrPres of synthetic origin is similar to abnormal C-terminal PK-resistant fragments found in most individuals with sporadic Creutzfeldt-Jakob disease, iatrogenic Creutzfeldt-Jakob disease, or mice infected with mouse-passaged hamster scrapie. ${ }^{33-35}$ Similar C-terminal PK-resistant fragments were found in atypical bovine spongiform encephalopathy and in certain types of ovine scrapie. ${ }^{36,37}$ In a report, co-accumulation of atypical PrPres and $\mathrm{PrP}^{\mathrm{Sc}}$ was reported in a patient who succumbed to sporadic Creutzfeldt-Jakob disease with a prolonged clinical course. ${ }^{38}$ Nevertheless, the relation between atypical PrPres of synthetic and natural origins has not yet been established. ${ }^{24}$

Previously infecting a host with two prion strains, one with a short and another with a long incubation time, was shown to lead to an extension of the incubation period to disease compared with the period for the short incubation time strain, a phenomenon known as prion strain interference. ${ }^{39-43}$ In the current study, we tested whether atypical PrPres can replace a long incubation time strain and interfere with replication of a short incubation time strain. Although transmissible, atypical PrPres does not produce prion disease ${ }^{8,10,12}$ and thereby might offer advantages as an interfering agent. In vitro, atypical PrPres and $\mathrm{PrP}^{\mathrm{Sc}}$ could be selectively amplified from their mixtures in two PMCAb formats via modification of the biochemical environment and the $\operatorname{PrP}^{\mathrm{C}}$ glycosylation status. ${ }^{17}$ Unfortunately, mixing atypical PrPres with the short incubation time strain $263 \mathrm{~K}$ did not delay the incubation time to the disease. There are several possible explanations for the lack of interference. Similar to $\mathrm{PrP}^{\mathrm{Sc}}$, atypical PrPres undergoes clearance after inoculation. Accumulation of atypical PrPres after clearance might occur slower than accumulation of the short incubation time strains, such as $263 \mathrm{~K}$, leading to a development of TSE before atypical PrPres had a chance to accumulate. In addition, atypical PrPres might not be effective as an interfering agent because of differences in neurotropism between atypical PrPres and $263 \mathrm{~K}$ and/or differences in subcellular replication sites. Deposition of atypical PrPres in different brain regions was found to overlap only partially with that of natural or synthetic prion strains. ${ }^{12}$ Unlike $\mathrm{PrP}^{\mathrm{Sc}}$, atypical PrPres did not show perineuronal or perivascular immunoreactivity. ${ }^{12}$ If the replication rates of $\mathrm{PrP}^{\mathrm{Sc}}$ and atypical PrPres were not controlled exclusively by a substrate but also availability of state-specific cellular cofactors as well, differences in cellular cofactors might contribute too to the apparent lack of an interference effect. ${ }^{17}$ Failure to delay the disease could be due to differences in the experimental design between the current and previous studies. In previous studies the long incubation 
time strains were inoculated much earlier than the short incubation time strains, allowing the long incubation time strains more time to spread through cellular replication sites. ${ }^{39-43}$ Finally, the most recent studies that used twodimensional analysis of PrP sialoglycoforms revealed that only a small subfraction of the $\operatorname{PrP}^{\mathrm{C}}$ that is recruited as a substrate into $\operatorname{PrP}^{\mathrm{Sc}}$ could also serve as a substrate for atypical PrPres. ${ }^{44}$ This explains why atypical PrPres replicates slower than $\operatorname{PrP}^{\mathrm{Sc}}$.

\section{Conclusions}

In previous studies, a mechanistic model for genesis of authentic $\operatorname{PrP}^{\mathrm{Sc}}$ from noninfectious fibrils that involves two main steps was established. ${ }^{8,10}$ The current study tested whether expression levels of substrate play a role in evolution of synthetic prions. We found that overexpression of $\operatorname{PrP}^{\mathrm{C}}$ facilitated the first step toward $\operatorname{PrP}^{\mathrm{Sc}}$, ie, the transition from $\mathrm{rPrP}$ fibrils to atypical PrPres. However, the second step toward $\operatorname{PrP}^{\mathrm{Sc}}$, which is controlled by the rate of deformed templating events, did not depend on the expression levels of substrate. These results suggest that the intrinsic rate of conformational errors in templating alternative self-propagating states is independent of substrate concentration. Nevertheless, overexpressing $\operatorname{PrP}^{\mathrm{C}}$ helped to expand the range of self-replicating states generated in vitro that are capable of inducing TSEs in animals. In addition, the current work explored the potential of atypical PrPres to interfere with replication of a short incubation time prion strain. Atypical PrPres was found to interfere effectively with replication of short incubation time strain $263 \mathrm{~K}$ in vitro; however it did not delay prion disease in animals inoculated with $263 \mathrm{~K}$.

\section{Acknowledgment}

We thank Pamela Wright for editing the manuscript.

\section{Supplemental Data}

Supplemental material for this article can be found at http://dx.doi.org/10.1016/j.ajpath.2015.11.013.

\section{References}

1. Prusiner SB: Prion diseases and the BSE crisis. Science 1997, 278: 245-251

2. Cohen FE, Prusiner SB: Pathologic conformations of prion proteins. Annu Rev Biochem 1998, 67:793-819

3. Prusiner SB, Scott MR: Genetics of prions. Annu Rev Genet 1997, 31 : 139-175

4. Sigurdson CJ, Nilsson KP, Hornemann S, Heikenwalder M, Manco G, Schwartz P, Ott D, Rulicke T, Liberski T, Julius C, Falsiq J, Stitz L, Wuthrich K, Aguzzi A: De novo generation of a transmissible spongiform encephalopathy by mouse transgenesis. Proc Natl Acad Sci U S A 2009, 106:304-309
5. Jackson WS, Borkowski AW, Faas H, Steele AD, King OD, Watson N, Jasanoff A, Lindquist S: Spontaneous generation of prion infectivity in fatal familial insomnia knockin mice. Neuron 2009, 63: $438-450$

6. Makarava N, Baskakov IV: Genesis of transmissible protein states vie deformed templating. Prion 2012, 6:252-255

7. Makarava N, Baskakov IV: The evolution of transmissible prions: the role of deformed templating. PLoS Pathog 2013, 9:e1003759

8. Makarava N, Kovacs GG, Savtchenko R, Alexeeva I, Budka H, Rohwer RG, Baskakov IV: Genesis of mammalian prions: from noninfectious amyloid fibrils to a transmissible prion disease. PLoS Pathog 2011, 7:e1002419

9. Makarava N, Kovacs GG, Bocharova OV, Savtchenko R, Alexeeva I, Budka H, Rohwer RG, Baskakov IV: Recombinant prion protein induces a new transmissible prion disease in wild-type animals. Acta Neuropathol 2010, 119:177-187

10. Makarava N, Kovacs GG, Savtchenko R, Alexeeva I, Ostapchenko VG, Budka H, Rohwer RG, Baskakov IV: A new mechanism for transmissible prion diseases. J Neurosci 2012, 32: 7345-7355

11. Makarava N, Kovacs GG, Savtchenko R, Alexeeva I, Budka H, Rohwer RG, Baskakov IV: Stabilization of a prion strain of synthetic origin requires multiple serial passages. J Biol Chem 2012, 287: 30205-30214

12. Kovacs GG, Makarava N, Savtchenko R, Baskakov IV: Atypical and classical forms of the disease-associated state of the prion protein exhibit distinct neuronal tropism, deposition patterns, and lesion profiles. Am J Pathol 2013, 183:1539-1547

13. Wan W, Bian W, McDonald M, Kijac A, Wemmer DE, Stubbs G: Heterogeneous seeding of a prion structure by a generic amyloid form of the fungal prion-forming domain HET-s(218-289). J Biol Chem 2013, 288:29604-29612

14. Atarashi R, Moore RA, Sim VL, Hughson AG, Dorward DW, Onwubiko HA, Priola SA, Caughey B: Ultrasensitive detection of scrapie prion protein using seeded conversion of recombinant prion protein. Nat Methods 2007, 4:645-650

15. Colby DW, Zhang Q, Wang S, Groth D, Legname G, Riesner D, Prusiner SB: Prion detection by an amyloid seeding assay. Proc Natl Acad Sci U S A 2007, 104:20914-20919

16. Makarava N, Ostapchenko VG, Savtchenko R, Baskakov IV: Conformational switching within individual amyloid fibrils. J Biol Chem 2009, 284:14386-14395

17. Makarava N, Savtchenko R, Baskakov IV: Selective amplification of classical and atypical prions using modified protein misfolding cyclic amplification. J Biol Chem 2013, 288:33-41

18. Breydo L, Sun Y, Makarava N, Lee CI, Novitskaia V, Bocharova OV, Kao JP, Baskakov IV: Nonpolar substitution at the C-terminus of the prion protein, a mimic of the glycosylphosphatidylinositol anchor, partially impairs amyloid fibril formation. Biochemistry 2007, 46:852-861

19. Kercher L, Favara C, Chan CC, Race R, Chesebro B: Differences in scrapie-induced pathology of the retina and brain in transgenic mice that express hamster prion protein in neurons, astrocytes, or multiple cell types. Am J Pathol 2004, 165:2055-2067

20. Bocharova OV, Breydo L, Parfenov AS, Salnikov VV, Baskakov IV: In vitro conversion of full-length mammalian prion protein produces amyloid form with physical property of $\operatorname{PrP}(\mathrm{Sc})$. J Mol Biol 2005, 346: 645-659

21. Committee for the Update of the Guide for the Care and Use of Laboratory AnimalsNational Research Council: Guide for the Care and Use of Laboratory Animals. ed 8. Washington, DC, National Academies Press, 2011

22. Gonzalez-Montalban N, Makarava N, Ostapchenko VG, Savtchenko R, Alexeeva I, Rohwer RG, Baskakov IV: Highly efficient protein misfolding cyclic amplification. PLoS Pathog 2011, 7:e1001277

23. Gonzalez-Montalban N, Makarava N, Savtchenko R, Baskakov IV: Relationship between conformational stability and amplification efficiency of prions. Biochemistry 2011, 50:7933-7940 
24. Klimova N, Makarava N, Baskakov IV: The diversity and relationship of prion protein self-replicating states. Virus Res 2015, 207:113-119

25. Deleault NR, Walsh DJ, Piro JR, Wang F, Wang X, Ma J, Rees JR, Supattapone S: Cofactor molecules maintain infectious conformation and restrict strain properties in purified prions. Proc Natl Acad Sci U S A 2012, 109:E1938-E1946

26. Legname G, Baskakov IV, Nguyen HO, Riesner D, Cohen FE, DeArmond SJ, Prusiner SB: Synthetic mammalian prions. Science 2004, 305:673-676

27. Colby DW, Giles K, Legname G, Wille H, Baskakov IV, DeArmond SJ, Prusiner SB: Design and construction of diverse mammalian prion strains. Proc Natl Acad Sci U S A 2009, 106:20417-20422

28. Colby DW, Wain R, Baskakov IV, Legname G, Palmer CG, Nguyen HO, Lemus A, Cohen FE, DeArmond SJ, Prusiner SB: Protease-sensitive synthetic prions. PLoS Pathog 2010, 6:e1000736

29. Ghaemmaghami S, Watts JC, Nquyen HO, Hayashi S, DeArmond SJ, Prusiner SB: Conformational transformation and selection of synthetic prion strains. J Mol Biol 2011, 413:527-542

30. Ghaemmaghami S, Colby DW, Nquyen HO, Hayashi S, Oehler A, DeArmond S, Prusiner SB: Convergent replication of mouse synthetic prion strains. Am J Pathol 2013, 182:866-874

31. Wille H, McDonald M, Bian W, Kendall A, Borovinskiy A, Cohen F, Prusiner S, Stubbs G: X-ray fiber diffraction reveals major structural differences between brain-derived prions and recombinant prion protein amyloid. Oct 8-10, 2008, Madrid, Spain, International Conference Prion-2008, 2008, Abstract 39

32. Ostapchenko VG, Sawaya MR, Makarava N, Savtchenko R, Nilsson KP, Eisenberg D, Baskakov IV: Two amyloid states of the prion protein display significantly different folding patterns. J Mol Biol 2010, 400:908-921

33. Zou WQ, Capellari S, Parchi P, Sy MS, Gambetti P, Chen SG: Identification of novel proteinase K-resistant C-terminal fragments of PrP in Creutzfeldt-Jakob disease. J Biol Chem 2003, 278:40429-40436

34. Satoh K, Muramoto T, Tanaka T, Kitamoto N, Ironside JW, Nagashima K, Yamada M, Sato T, Mohri S, Kitamoto T: Association of an 11-12 $\mathrm{kDa}$ protease-resistant prion protein fragment with subtypes of dura graft-associated Creutzfeldt-Jakob disease and other prion diseases. J Gen Virol 2003, 84:2885-2893

35. Lawson VA, Priola SA, Meade-White K, Lawton M, Chesebro B Flexible N-terminal region of prion protein influences conformation of protease-resistant prion protein isoforms associated with cross-species scrapie infection in vivo and in vitro. J Biol Chem 2004, 279: 13689-13695

36. Biacabe AG, Jacobs JG, Bencsik A, Langeveld JP, Baron TG: H-type bovine spongiform encephalopathy: complex molecular features and similarities with human prion diseases. Prion 2007, 1:61-68

37. Baron T, Bencsik A, Vulin J, Biacabe AG, Morignat E, Verchere J, Betemps D: A C-terminal protease-resistant prion fragment distinguishes ovine "CH1641-like" scrapie from bovine classical and LType BSE in ovine transgenic mice. PLoS Pathog 2008, 4:e1000137

38. Rodrigues-Martinez AB, de Munain AL, Ferrer I, Zarranz JJ, Atares B, Villagra NT, Arteagoitia JM, Garrido JM, Juste RA: Coexistence of protease sensitive and resistant prion protein in 129VV homozygous sporadic Creutzfeldt-Jakob disease: a case report. J Med Case Rep 2012, 6:348

39. Dickinson AG, Fraser H, Meikle VM, Outram GW: Competition between different scrapie agents in mice. Nat New Biol 1972, 237: 244-245

40. Kimberlin RH, Walker CA: Competition between strains of scrapie depends on the blocking agent being infectious. Intervirology 1985 , 23:74-81

41. Bartz JC, Kramer ML, Sheehan MH, Hutter JA, Ayers JI, Bessen RA, Kincaid AE: Prion interference is due to a reduction in strain-specific PrPSc levels. J Virol 2007, 81:689-697

42. Schutt CR, Bartz JC: Prion interference with multiple prion isolates. Prion 2008, 2:61-63

43. Shikiya RA, Ayers JI, Schutt CR, Kincaid AE, Bartz JC: Coinfecting prion strains compete for a limiting cellular resource. J Virol 2010, 84: $5706-5714$

44. Makarava N, Savtchenko R, Baskakov IV: Two alternative pathways for generating transmissible prion disease de novo. Acta Neuropathol Commun 2015, 3:69 